Ann. Zootech., I968, 17 (I), 47-57.

\title{
RÉSULTATS OBTENUS AU COURS DE L'ÉTUDE DE QUELQUES FACTEURS IMPORTANTS DE L'INSÉMINATION ARTIFIGIELLE OVINE
}

\author{
G. COLAS, L. DAUZIER, M. COUROT, R. ORTAVANT, J.-P. SIGNORET \\ Laboratoire de Physiologie de la Reproduction, \\ Centre de Recherches vétérinaires et zootechniques, 37 -Nouzilly \\ Laboraloire de Zootechnie, \\ École nationale supérieure agronomique, \\ Centre de Recherches agronomiques du Midi, 34-Montpellier \\ Institut national de la Recherche agronomique
}

\section{SOMMAIRE}

La recherche d'une technique d'insémination artificielle dans l'espèce ovine a été orientée vers l'étude du milieu de dilution, de la durée et de la température de conservation du sperme et du nombre quotidien d'interventions. Au cours de nos expériences, I 598 brebis de races Lacaune et lle-de-France ont été utilisées.

I' Une comparaison entre le lait de vache écrémé et le cuE a révélé une différence significative en faveur du premier dilueur $(75,4 \mathrm{p}$. Ioo de mise bas contre $66,4 \mathrm{p}$. Ioo après insémination pendant une seule période d'œestrus, $\mathrm{P}<0,0 \mathrm{I})$.

$2^{\circ}$ Dans ces conditions, la température de $+15^{\circ} \mathrm{C}$ assure une préservation satisfaisante du pouvoir fécondant de la semence pendant les quatorze premières heures.

Par contre, le refroidissement à $+4^{\circ} \mathrm{C}$ entraîne une baisse importante de fécondance mais celle-ci ne s'accuse pas beaucoup au cours de la conservation.

$3^{0} \mathrm{La}$ fertilité augmente significativement avec la fréquence journalière d'interventions (I I. A./j : 5I,7 p. roo de mise bas ; 2 I. A./j:64,6 p. 100; $\mathrm{P}<0,05)$ et la durée des chaleurs (6I p. Ioo de mise bas pour les durées d'œestrus inférieures à $24 \mathrm{~h}, 76,5 \mathrm{p}$. Ioo pour les autres; $\mathrm{P}<0,0 \mathrm{I}$ ).

Des différences significatives ont été observées entre les niveaux de fécondance des différents béliers $(39,4$ p. 100 à 76,7 p. 100).

Avec la meilleure méthode, nous avons enregistré un taux de mise bas supérieur à $75 \mathrm{p}$. Ioo. Ce résultat, comparable à celui que l'on peut obtenir avec la saillie naturelle, justifie l'exploitation, dans le domaine pratique, de la technique d'insémination artificielle.

\section{INTRODUCTION}

L'insémination artificielle chez les Ovins est largement répandue dans certains pays possédant de très grands troupeaux. Cependant, les résultats publiés sont parfois discordants et il reste encore de nombreux problèmes à résoudre avant d'envisager 
l'application de cette technique dans un pays comme la France dont la structure de l'élevage est faite essentiellement de petites unités dispersées.

Si l'utilisation immédiate de la semence pure ou peu diluée donne des résultats très satisfaisants (KARDIMOWICZ, I953; I)AUZIER et al., I954 ; SESERMAN et al., I962 ; LuxCA et al., I962 ; IVAKNENKo et RAK, I964), la conservation du sperme s'accompagne généralement d'une baisse du taux de fertilité (SALAmon et RoBrNsox, I 962 b). La littérature abonde en travaux dans ce domaine mais rares sont les publications qui donnent des indications précises. Ie plus, les techniques rapportées sont très diverses, (DAuzier, I956 ; Habibuins, I963 ; Bei,JAkov, I964; Lunca et al., I $964 \ldots)$.

Or, l'intérêt présenté par l'insémination artificielle ovine a pris récemment des dimensions nouvelles en raison de l'utilisation des progestagènes pour la synchronisation des chaleurs et de la nécessité, dans le cas du testage, de diffuser la semence des béliers de la manière la plus large possible. Nous avons donc été amenés à reprendre cette étude dans son ensemble. Le présent article rend compte des essais entrepris depuis 1964 .

\section{MATÉRIEL ET MÉTHODES}

Quatre expériences ont été réalisées au cours des années I964 à 1966 sur un ensemble de I 598 animaux : elles sont résumées dans le tableau I.

\section{Animaux expérimentaux}

Les brebis, âgées de $\mathbf{~}$ à 8 ans, appartenaient au troupeau expérimental du laboratoire (race Ilede-France) ou à des éleveurs privés (race Lacaune, région du Larzac). Dans tous les cas, l'apparition des chaleurs était spontanée.

Selon les essais, 4 à 6 béliers de r à 4 ans et demi d'âge ont été utilisés. Aucun d'eux n'avait fait l'objet d'un entrainement préalable à la collecte au vagin artificiel.

\section{Dilueurs}

Au cours des deux prenières expériences, deux milieux de conservation ont été utilisés : le lait de vache écrémé reconstitué et le CUE (FoOTE, I958). Lors de sa préparation, le lait reconstitué ( $10 \mathrm{~g}$ de poudre $+90 \mathrm{ml}$ d'eau distillée $+300 \mathrm{mg}$ de sulfanides) était maintenu pendant เo minutes à une température de $90^{\circ} \mathrm{C}$ environ, puis refroidi $\left(25-30^{\circ} \mathrm{C}\right.$ ) et additionné d'antibiotiques ( $10^{5}$ UI pénicilline et $100 \mathrm{mg}$ streptomycine pour la quantité préparée). Le cue préparé chaque jour à partir de tampon salin et de jaune d'ouf frais, recevait des antibiotiques dans les mêmes proportions. De la catalase $(3000 \mathrm{UI} / \mathrm{mg})$ était incorporée aux dilueurs, à raison de $20 \mu \mathrm{g} / \mathrm{ml}$ de sperme dilué (HAFs, 196r ; FoOTE, 1962). Ultérieurement, seul le dilueur lait + catalase a été utilisé (tabl. i).

\section{Récolle et dilution de la semence}

Les récoltes avaient lieu une fois par jour : un à trois éjaculats par bélier étaient collectés à l'aide d'un vagin artificiel et aussitôt placés dans un bain-marie $\left(3 \mathrm{O}^{\circ} \mathrm{C}\right)$. La dilution suivait immédiatement le contrôle de motilité et la mesure photocolorimétrique de la concentration. Le dilueur était ajouté à la semence dans le rapport 3/I dans la première expérience ou de manière à parvenir à une concentration finale de $625 \times 10^{6}$ spermatozoides par $\mathrm{ml}$ dans les autres.

\section{Refroidissement et conservation de la semence}

Après dilution, le sperme était refroidi $\mathfrak{a}+I^{\circ} 5^{\circ}$ en $z^{\circ}$ minutes environ ou $\grave{a}+4^{\circ} \mathrm{C}$ en $\mathrm{I} h 4^{\circ}$ et maintenu à ces températures par des ampoules d'acide acétique ou de glace en fusion. La vitesse de refroidissement avait été déterminée au cours d'une étude préalable réalisée au laboratoire. 
INSÉMINATION AR'TIFICIELLE OVINE

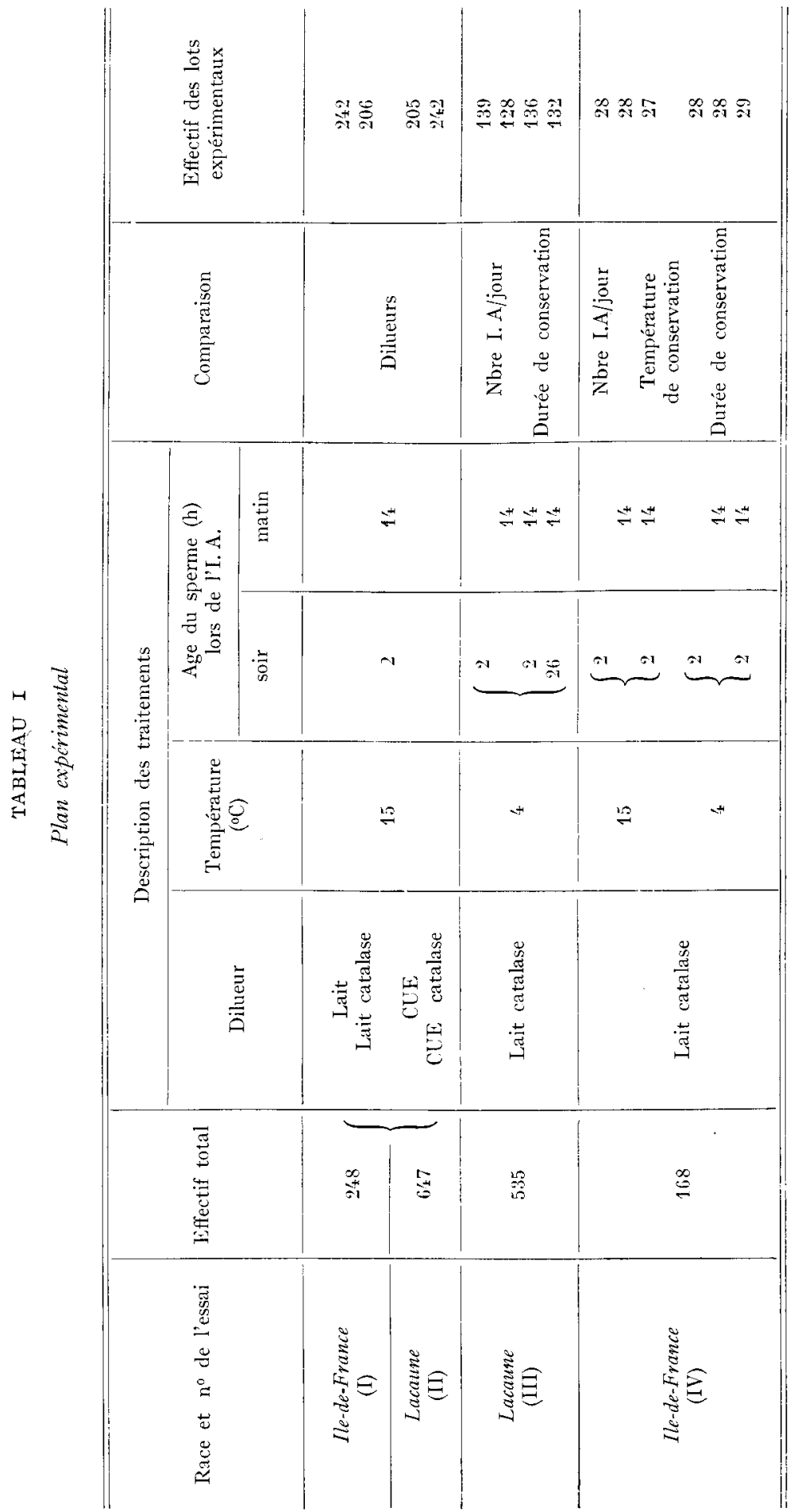




\section{Contrôles de la semence}

La motilité d'ensemble était notée de o à 5 . La majorité des éjaculats retenus présentaient une motilité supérieure ou égale à 4,5 . A ce premier examen, s'ajoutaient les mesures du volume et de la concentration en spermatozoïdes pour l'ajustement de la dilution.

Le sperme dilué faisait l'objet d'un nouveau contrôle (motilité individuelle et pourcentage de spermatozoïdes vivants) avant d'être utilisé.

\section{Insémination}

La détection des chaleurs était assurée une fois par jour par des béliers équipés de tablier. Les inséminations étaient répétées pendant toute la durée des chaleurs, une ou deux fois par jour, selon les essais (tabl. I).

$0,2 \mathrm{ml}$ de semence étaient mis en place à chaque insémination, ce qui, dans les trois derniers essais, correspondait à $125 \cdot 10^{6}$ spermatozoïdes par intervention. Le sperme était déposé au fond du vagin à l'aide d'un spéculum tubulaire éclairant dans l'essai I, ou dans l'entrée du cervix grâce à un spéculum gynécologique dans les essais suivants.

\section{Analyse des résultats}

Ces essais ont été conduits selon les principes de l'analyse factorielle pour les variables considérées (béliers, élevages, traitements) avec permutation régulière de chacune d'elles plusieurs fois au cours d'une même période expérimentale.

Les données sont analysées selon la méthode $\chi^{2}$ de PEArson.

\section{RÉSULTATS}

L,es résultats sont exprimés en taux de mise bas calculés après insémination pendant un cestrus.

\section{Comparaison des dilueurs}

L a comparaison des résultats obtenus avec les différents dilueurs fait apparaître un écart hautement significatif en faveur du lait $(\mathrm{P}<0,0 \mathrm{r}$, tabl. 2).

TABLEAU 2

Influence du dilueur, additionné ou non de calalase, sur le taux de mise bas des brebis inséminćes artificiellement au cours d'un a'sirus

\begin{tabular}{|c|c|c|c|}
\hline \multirow{2}{*}{ Dilueur } & \multicolumn{2}{|c|}{ Catalase } & \multirow{2}{*}{ Total } \\
\hline & + & 0 & \\
\hline Lail & $\begin{array}{c}7+6 \% \\
(206)\end{array}$ & $\begin{array}{c}76,0 \text { a } \\
(2 \div 2 \div)\end{array}$ & $\begin{array}{c}75, y^{\prime} \% \\
(1+4)\end{array}$ \\
\hline CLF: & $\begin{array}{c}69, y^{\prime} \% \\
\left(y^{\prime}+2\right)\end{array}$ & $\begin{array}{c}62,9 \% \\
(205)\end{array}$ & $\begin{array}{c}66,4 \% \\
(447)\end{array}$ \\
\hline l'otal & $\begin{array}{c}71,8 \% \\
(1+8)\end{array}$ & $\begin{array}{c}70,0 \% \\
\left(x^{\prime} 7\right)\end{array}$ & $\begin{array}{l}71,0 \% \\
(895)\end{array}$ \\
\hline
\end{tabular}

( ) nombre de brebis inséminées. 
La catalase n'améliore, d'une manière significative, aucun des milieux de conservation mais, dans l'ensemble, son effet paraît plutôt favorable. C'est pourquoi nous l'avons utilisée dans les trois derniers essais (tabl. 2).

\section{Durée de conservation}

Dans les deux premiers lots (tabl. 3, exp. III et IV), les interventions ont été réalisées à des moments différents par rapport au début observé des chaleurs : le soir, c'està-dire dès le contrôle de l'œstrus ou le lendemain matin. L'effet d'une courte durée de conservation ( $\mathrm{I}_{4} \mathrm{~h}$ ) n'apparaît donc pas ici à l'état pur.

TABLEAU 3

Influence de la durée de conservation sur le pouvoir fécondant du sperme de Bélier

\begin{tabular}{|c|c|}
\hline Durée de conservation & Taux de mises bas \\
\hline $\begin{array}{l}\because \text { heures } \\
11 \text { heures }\end{array}$ & $\begin{array}{c}52,3 \\
(195) \\
51,1 \\
(184)\end{array}$ \\
\hline $\begin{array}{l}2 \text { et } 1 ' \text { heures } \\
1 ' t \text { et } 26 \text { heures }\end{array}$ & $\begin{array}{c}6.4,0 \\
(136) \\
58,3 \\
(132)\end{array}$ \\
\hline
\end{tabular}

() Nombre d'animaux inséminés.

Il semble cependant que le pouvoir fécondant des spermatozoïdes diminue légèrement au cours de la conservation, comme l'indiquent les résultats obtenus dans les lots suivants $(64,0$ p. roo contre $58,3 \mathrm{p}$. roo). Cette influence dépressive s'exerce également sur leur survie (fig. I).

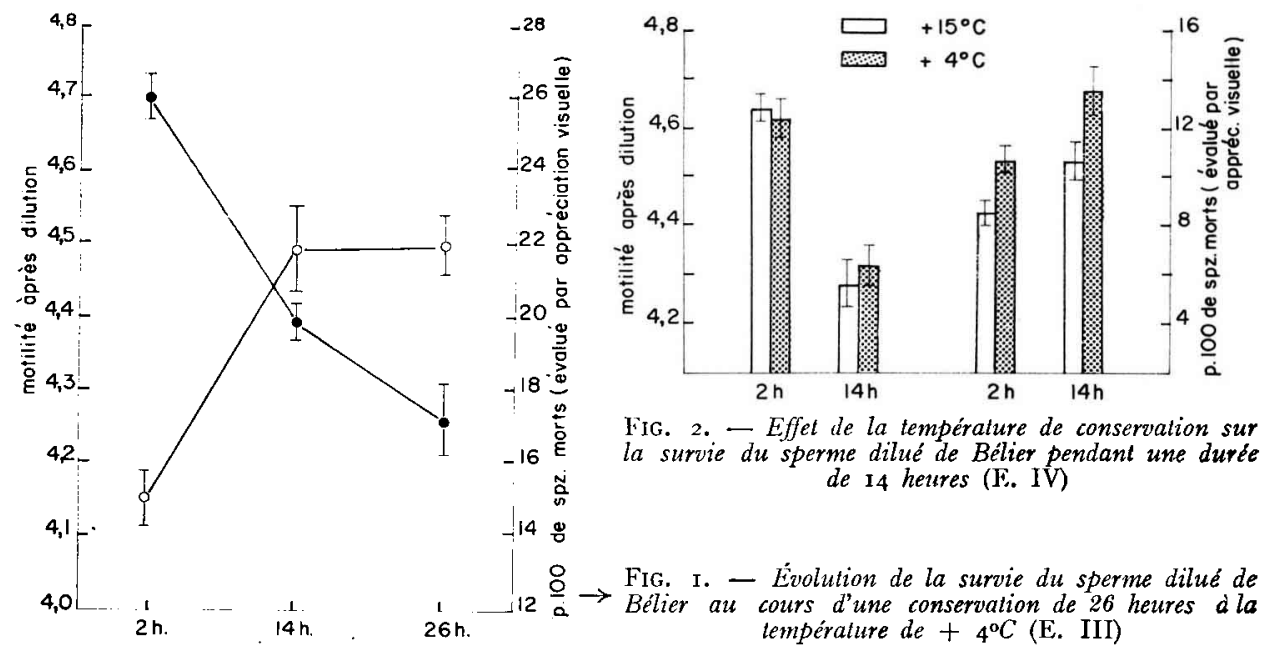




\section{Température de conservation}

Le sperme conservé à la température de $+5^{\circ} \mathrm{C}$ présente, chez tous les béliers, le pourcentage le moins élevé de spermatozoïdes morts (fig. 2).

Les écarts dans les taux de mise bas ne sont pas significatifs dans l'essai IV $\left(58,8\right.$ p. roo de M. B. à I $5^{\circ} \mathrm{C}, 53,0$ p. Ioo à $\left.4^{\circ} \mathrm{C}\right)$. Néanmoins, la comparaison des résultats enregistrés dans les expériences II et III fait ressortir nettement l'effet défavorable de la température $+4^{\circ} \mathrm{C}$.

\section{Nombre d'inséminations par jour}

Comme l'indique le tableau 4 , la fertilité augmente avec la fréquence journalière d'intervention $(\mathrm{P}<0,05)$. Toutefois, il faut préciser que le nombre quotidien de spermatozoïdes déposés n'était pas identique dans les deux cas ( $125 \times 10^{6}$ contre $\left.250 \times 10^{6}\right)$. Il est donc difficile de dissocier l'influence respective de ces deux facteurs.

\section{TABREAU 4}

Infuence de la fréquence d'insémination sur la fertilití des brebis après un seul restrus (Lssais III et IV)

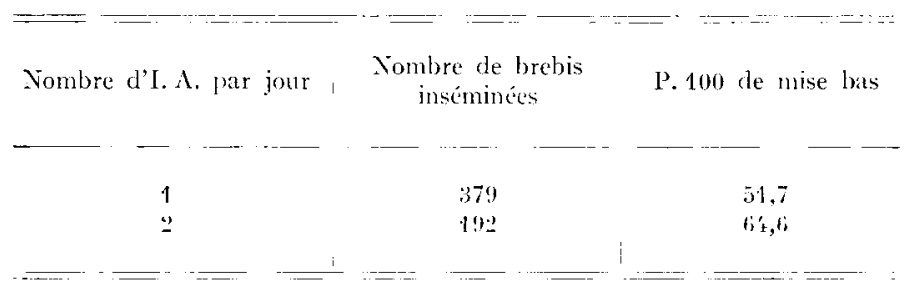

5. Durée d'xestrus

Quelle que soit la race, le taux de mise bas est toujours plus élevé $(\mathrm{P}<0,0 \mathrm{I})$ chez les animaux dont la durée d'œstrus est supérieure ou égale à 24 heures (tabl. 5).

TABLEAU 5

Influence de la durée d'astrus sur le tanx de mise bas des brebis inséminées artificiellement au cours d'un a'strus

(Essais I et II)

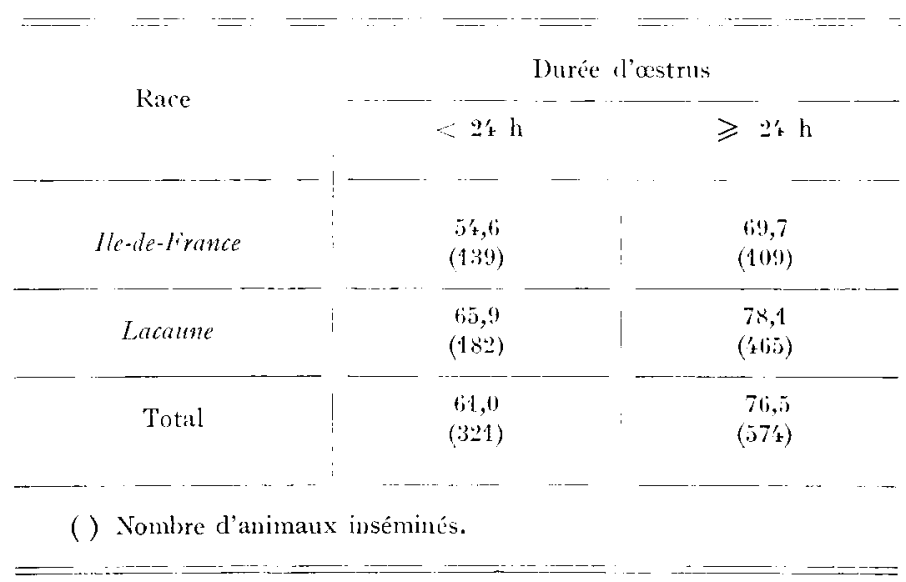




\section{6. Élevages}

Les troupeaux de race Lacaune communs aux deux essais ont été réunis d'après leur niveau de fertilité (tabl. 6). Il est intéressant de noter que la supériorité des élevages du premier groupe apparaît dans les résultats de chacune des expériences entreprises, quels que soient les traitements appliqués.

\section{TABLEAU 6}

Différences de fertilité observées entre trois troupeaux de race Lacaune, déterminées d'après le taux de mise bas des brebis inséminées artificiellement au cours d'un astrus

\begin{tabular}{|c|c|c|}
\hline Essai & Élevages $A$ et $B$ & Élevages $\mathrm{C}$ et $\mathrm{D}$ \\
\hline II & $\begin{array}{c}78,2 \\
(170)\end{array}$ & $\begin{array}{c}69,1 \\
(210)\end{array}$ \\
\hline III & $\begin{array}{r}63,0 \\
(293)\end{array}$ & $\begin{array}{r}47,6 \\
(189)\end{array}$ \\
\hline Total & $\begin{array}{c}69,2 \\
(13)\end{array}$ & $\begin{array}{c}58,9 \\
(399)\end{array}$ \\
\hline
\end{tabular}

( ) Nombre d'animaux inséminés.

\section{Béliers}

Le tableau 7 fait ressortir des différences de fécondance élevées entre béliers $(\mathrm{P}<\mathrm{o}, \mathrm{or})$. Toutefois, ces écarts n'ont pas la même importance dans toutes les expériences. Dans les deux premiers essais, en particulier, ils ne sont pas significatifs.

On trouve aussi d'importantes variations dans les caractéristiques séminales des béliers. Il est curieux de constater, par exemple, que les deux animaux dont le volume de sperme et la quantité de spermatozoïdes par éjaculat ont les valeurs les plus faibles, sont les moins féconds (béliers $n^{\circ} 3$ et 6 ). Les interventions avaient pourtant été réalisées, dans tous les cas, avec un nombre identique de spermatozoïdes.

\section{DISCUSSION}

\section{Milieu et température de conservation}

Les taux de fertilité (essais I et II) enregistrés avec le C. U. E. demeurent assez constants $(65,7 \mathrm{p}$. Ioo contre $68,5 \mathrm{p}$. I00) bien que, dans l'expérience II, la technique de mise en place ait été plus précise et le nombre moyen d'interventions par animal supérieur $(2,9$ chez les brebis Ile-de-France contre 3,7 chez les Lacaune). Par contre, il existe des écarts sensibles entre les résultats obtenus avec le dilueur au lait écrémé 


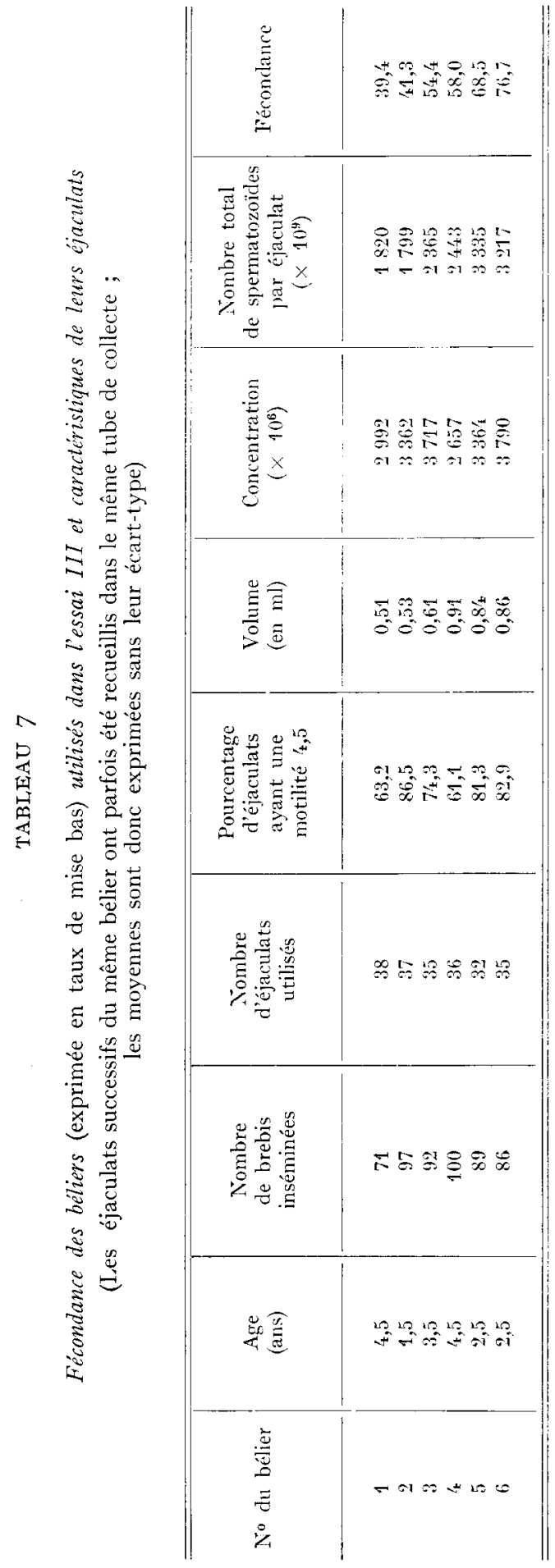


et, dans les meilleurs lots, le pourcentage de mise bas atteint des valeurs comparables à celles que l'on rencontre en saillie naturelle.

S'il est vrai que le lait écrémé constitue un bon dilueur, il faut souligner que ses qualités protectrices à l'égard de la semence dépendent de la température. Utilisé à $\div 4^{\circ} \mathrm{C}$, il n'assure plus un aussi bon maintien du pouvoir fécondant (Salamov et Robinson, I 962 a). Dans nos essais in vitro, cette action défavorable du refroidissement à $+4^{\circ} \mathrm{C}$ n'apparaît que dans le pourcentage de spermatozoïdes vivants. Il s'y ajoute vraisemblablement une modification irréversible du métabolisme cellulaire, causée par l'abaissement de la température du sperme à $+5^{\circ} \mathrm{C}$ (O'SHEA et WALES, I966).

\section{Durée de conservation}

De nombreux auteurs ('Terrili, I940 ; DaUzIER, I956 ; SALAmon et Robinson, I $962 b$ ) constatent chez le Bélier une chute importante du pouvoir fécondant dès les premières 24 heures de conservation. Notre expérience ne confirme pas entièrement ces observations, mais elle fait tout de même apparaître un léger effet dépressif (non significatif) de cette durée de conservation sur les qualités fécondantes des spermatozoïdes.

\section{Durée d'cestrus}

Comme nous l'avons montré (tabl. 7), la fertilité de la Brebis augmente avec la durée de l'œstrus. Toutefois, nos expériences ne permettent pas de préciser, si cette élévation du taux de conception est due à un plus grand nombre d'interventions $(2,4$ ou 6$)$ ou de spermatozoïdes déposés $\left(250,500\right.$, ou1 $\left.750 \times 10^{6}\right)$.

\section{Béliers}

Le pouvoir fécondant du sperme de bélier peut difficilement être relié à sa motilité lorsque celle-ci a déjà fait l'objet d'une sélection rigoureuse. Cependant, dans ces conditions, l'examen du volume et du nombre total de spermatozoïdes des éjaculats reflète peut-être la valeur reproductive d'un animal, comme semblent l'indiquer nos résultats (tabl. 7). Si elle se confirmait, une telle relation signifierait qu'une augmentation de la production de sperme s'accompagne chez le Bélier d'une amélioration de sa qualité.

\section{CONCLUSION}

Les résultats obtenus au cours de ces expériences montrent que la meilleure technique consiste à utiliser du sperme dilué dans du lait écrémé et maintenu à la température de $+\mathrm{r}_{5}^{\circ} \mathrm{C}$ pendant une durée maximum de $\mathrm{r}_{4}$ heures. Si cette méthode doit encore être améliorée, notamment en ce qui concerne la conservation de la semence, elle peut dès maintenant être utilisée avec succès dans le domaine pratique et assurer un taux de mise bas supérieur à $75 \mathrm{p}$. Ioo après une seule période d'insémination. Des essais ultérieurs ont d'ailleurs confirmé son efficacité. 


\title{
REMERCIEMEN'TS
}

Cette expérience a été réalisée grâce à l'aide de la Confédération générale des Producteurs de Lait de Brebis et des Industriels de Roquefort (M. Cottier et ses techniciens) et des éleveurs qui ont bien voulu accepter de mettre leurs troupeaux à notre disposition (MM. Bellas, Boudou, Camplo, Fabreguette, Rouquette et Singla). Nous tenons à leur exprimer tous nos remerciements. L'aide de techniciens de la Station centrale de Génétique animale de l'I. N. R. A. a été particulièrement appréciée. Nous remercions aussi la Société des Caves de Roquefort.

\section{SUMMARY}

\author{
EFFECT OF DILUTER, TEMPERATURE AND DURATION OF SPERM STORAGE, \\ AND FREQUENCY OF INSEMINATIONS ON FERTILITY OF EWES
}

Four experiments were undertaken in the fields on I 598 Lacaune and Ile-de-France ewes in order to study the effects of diluter, temperature and duration of sperm storage, and frequency of inseminations on lambing performance (table $\mathrm{r}$ ).

The ewes were inseminated with $0.2 \mathrm{ml}$ of semen, diluted to $\mathrm{I} / 4$ in the first experiment, and with constant number of spermatotozoa in the three other ones $\left(625 \times 10^{6}\right.$ per $\left.\mathrm{ml}\right)$. They were repeatedly inseminated for the whole time of heat.

I - In experiments $\mathrm{I}$ and 2 , two diluters were compared : skimmed cow milk (ro $\mathrm{g}$ of powder $+90 \mathrm{ml}$ of distilled water $+300 \mathrm{mg}$ of sulfamides) and $\mathrm{CLE}$; the two of them added with penicillin (I ooo IU per $\mathrm{ml}$ ) and streptomycin ( $\mathrm{r} \mathrm{mg}$ per $\mathrm{ml}$ ). The ewes were inseminated twice a day with semen stored at $\mathrm{I} 5^{\circ} \mathrm{C}$ for $\mathrm{I} 4$ hours.

The average lambing rate was higher with milk : 75.4 per cent, than with CLE $: 66.4$ per cent $(\mathrm{P}<0.01)$. The addition of catalase at $20 \gamma$ per $\mathrm{ml}$ of diluted semen resulted in a slight, not significant increase in fertility.

II - The use of sperm stored at $4^{\circ} \mathrm{C}$ resulted in a general decrease in fertility, confirmed with experiment 4 where the comparaison between the two temperatures was taken up as a factorial experiment. But the difference remained unsignificant : 58.8 per cent lambings at $\mathrm{I}_{5}{ }^{\circ} \mathrm{C}$ versus 53.0 at $4^{\circ} \mathrm{C}$.

A longer ( 26 hours) storage of sperm at $4^{\circ} \mathrm{C}$ did not significantly alter the lambing rate.

III - The lambing rate increased with the frequency of inseminations : $5 \mathrm{I} .7$ per cent for $\mathrm{I}$ insemination per day, v. 64.6 per cent for $2(P<0.05)$; and with the duration of oestrus : 61 .o per cent in ewes with a less than 24 hour heat duration, v. 76.5 for the other ones $(\mathrm{P}<0.0 \mathrm{I})$.

IV - There were differences in fertility between rams $(39.4$ to 76.7 per cent, $\mathrm{P}<0.0 \mathrm{I})$ and between breeds $(\mathrm{P}<0.0 \mathrm{r})$.

The highest lambing rate was more than 75 per cent, which equals natural mating. It could be reached with the following pattern :

milk + catalase diluter, I $25 \times 10^{6}$ spermatozoa per $0,2 \mathrm{ml}$ insemination, repeated twice a day. storage at $15^{\circ} \mathrm{C}$ for $\mathrm{I} 2$ to 15 hours.

\section{RÉFÉRENCES BIBLIOGRAPHIQUES}

Beljakov S. P., I964. Pourcentage de fécondation des brebis inséminées avec du sperme dilué et conservé à une température de $+2 \grave{a}+18^{\circ} \mathrm{C}$ (en russe). Ovtsevodstvo, 10 (1 $\left.\mathrm{r}\right)$, , $8-20$.

Dauzier L., Thibault C., Wintemberger S., i954. Conservation du sperme de bélier après dilution et maintien de son pouvoir fécondant. Ann. Endocrin., 15, 341-350.

Dauzier L., I956. Quelques résultats sur l'insémination artificielle des Brebis et des Chèvres en France. III $\mathrm{I}^{\mathrm{e}}$ Congr. Int. Reprod., Cambridge, I2-I4. 
Foote R. H., 1958. Fertility of bull semen stored for one and two days at $5^{\circ} \mathrm{C}$ in 20 p. 100 yolk citrateglycine-glucose extender. J. Dairy Sci., 41, 732 .

Foote R. H., 1962. Survival of bull sperm in milk and yolk extenders with added catalase. J. Dairy Sci. 45, 907-910.

Habibulin ig63. Conservation du sperme de Bélier à des températures supérieures à ooC (en russe). Ov/sevodstvo, $7,28-29$.

Hafs H. D., r961. Fertility of bull sperm with added catalase. J. Dairy Sci., 44, r529-I536.

IVAkhnenko V. K., RAK L. P., 1964. Durée de conservation du sperme de Bélier à une température nor male (en russe). Ovisevodstvo, $10(8)$, I I-I3.

Kardimowicz M., i953. Modycyne Weterynaryjne 9, 402.

LIson I., I958. Statistiques appliquées à la Biologie expérimentale. La planification de l'expérience el l'analyse des résultats. Gauthier-Villars édit.

Lunca N., Otel V., Paraschivescu M., Seserman O., 1961. Progresses realized by artificial insemination in Sheep with diluted semen. Proc. IVlh Int. Congr. Anim. Reprod. The Hague, 876-882.

Lunca N., Otel V., Paraschivescu M., Seserman O., Feredean T., Marsian A., Monnacs M., Georgescu D., Paunescu J, Ig62, Contributions à l'extension des inséminations artificielles avec le sperme dilué chez les Ovins. Lucrarile Stintifice ale institulului de cercetari soolehnice., 20, Editura agrosilvica.

O'Shea T., Wales R. C., I966. Effect of casein, lecithin, glycerol and storage at $5^{\circ} \mathrm{C}$ on diluted ram and bull semen. Austr. J. Biol. Sci., 19, $871-882$.

Salamon S., Robinson T. J., I962 a. Studies on artificial insemination of Merino Sheep. I. The effects of frequency and season of insemination, age of the ewe, rams and milk diluents on lambing performance. Aust. J. Agric. Res., 13, 52-68.

Salamon S., Rorinson T. J., $1962 b$. Studies on artificial insemination of Merino Sheep. II. The effects of semen dìluents and storage on lambing performance. Austr. J. Agric. Res., 13, 271-281.

Seserman O., Paraschivescu M., Lunca N., Orei V., ig62. Les propriétés du sperme et la fécondité des béliers Mérinos de Palas. Lucrârile Stintifice ale institutului de cercetari zoolehnice, 20, Editura agro silvicâ.

TERRIL C. E., I940. Comparison of ram semen collection obtained by three different methods for artificial insemination. Proc. Amer. Soc. Anim. Prod. 33rd. Ann. Mtg., 201-207. 